\title{
Complex Materials: The Tough Life of Bone
}

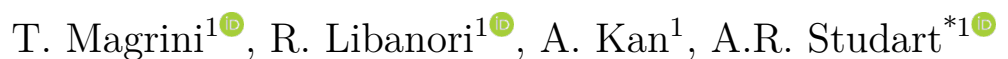 \\ ${ }^{1}$ Complex Materials, Department of Materials, ETH Zurich, 8093 Zurich, Switzerland.
}

Received on October 14, 2020. Revised on December 07, 2020. Accepted on December 08, 2020.

\begin{abstract}
Bone was a crucial biological material for the evolution of large terrestrial organisms and is today essential for most of our daily activities and well-being. From an engineering perspective, this living material features highly desirable properties for modern load-bearing structures. It is made of abundant and environmental-friendly building blocks, which are combined into a tough and durable structure that can continuously modify itself to adapt to changes in the mechanical load imposed by the surroundings. In this review article, we compile and discuss scientific findings that allow us to understand bone as a complex system with properties that emerge from cell-mediated interactions of molecules and particles at multiple length scales. Analogous to other complex systems, such interactions lead to self-organization, hierarchical structures and adaptive behavior without the need of a central controlling unit. A rich range of physical, chemical and biological phenomena provide a framework for information to be generated and processed in this complex system. Understanding the interplay between such underlying phenomena and their emerging properties should help the diagnosis and treatment of bone-related medical conditions and might provide guidelines for the future development of more sustainable materials and engineering structures.
\end{abstract}

Keywords: Complexity, Self-organisation, Hierarchical structures, Adaptation, Networks.

\section{Introduction}

From insect colonies to stock markets to the world wide web, complex systems are ubiquitous in nature, society and modern technologies [1]. In these systems, collective properties, information processing capabilities and adaptive response emerge from the interactions between a large number of individual constituents without a central controlling unit. Such features are prominent in living organisms, where complexity and diversity have been the natural outcome of billions of years of evolution. Complexity in living organisms derives from cell-mediated self-organization processes that create hierarchically structured systems capable of adapting to their environment. This holds true for a wide variety of living species from primitive $E$. coli bacteria to the Homo sapiens, despite the unclear role of natural selection on the increasing complexity observed [1]. While most of the organs and tissues of our body share these common attributes, bone is a remarkable example of an animal tissue where all these complex characteristics are combined with an unusual set of highly desirable material properties: low weight, stiffness and toughness.

Stiffness is the ability to carry mechanical load with minimum deformation and is the property that bone is mostly known for. Less intuitive is the strong resistance of bone against the propagation of cracks, which is referred to as fracture toughness. Stiffness and toughness provide the long-term mechanical stability

\footnotetext{
* Correspondence email address: andre.studart@mat.ethz.ch
}

required for locomotion and physical activities, whereas low weight is essential to minimize the energy needed to undertake such tasks. To fully appreciate the high mechanical stability of bone one should note that the main solid building blocks of this biological material, calcium phosphate and collagen, are intrinsically rather weak and soft, respectively. Indeed, the resistance of these mineral and organic building blocks against crack propagation is at least three-fold lower than that of the weakest engineering materials (Figure 1 a, b). Mixing these weak materials in the proportions found in bone would in principle lead to a mediocre fracture toughness. Remarkably, the hierarchical structure of bone provides mechanisms to break this rule and reach toughness levels comparable to those of engineering materials. It is important to emphasize that this is achieved without sacrificing the high stiffness of the calcium phosphate mineral phase. Combining high stiffness and toughness using weak building blocks is a hallmark that differentiates biological structural materials from their engineering counterparts, which typically show a trade-off between these essential mechanical properties (Figure 1a, b). The low strength of nature's building blocks is largely compensated by their wide availability and the fact that they can be processed into stiff and tough biological materials in water near room temperature. Such mild processing conditions are highly desirable but currently unmet in most manufacturing technologies.

The impressive toughness and stiffness of bone contrast with the fascinating dynamic and living nature of this biological material. Such living character is often 

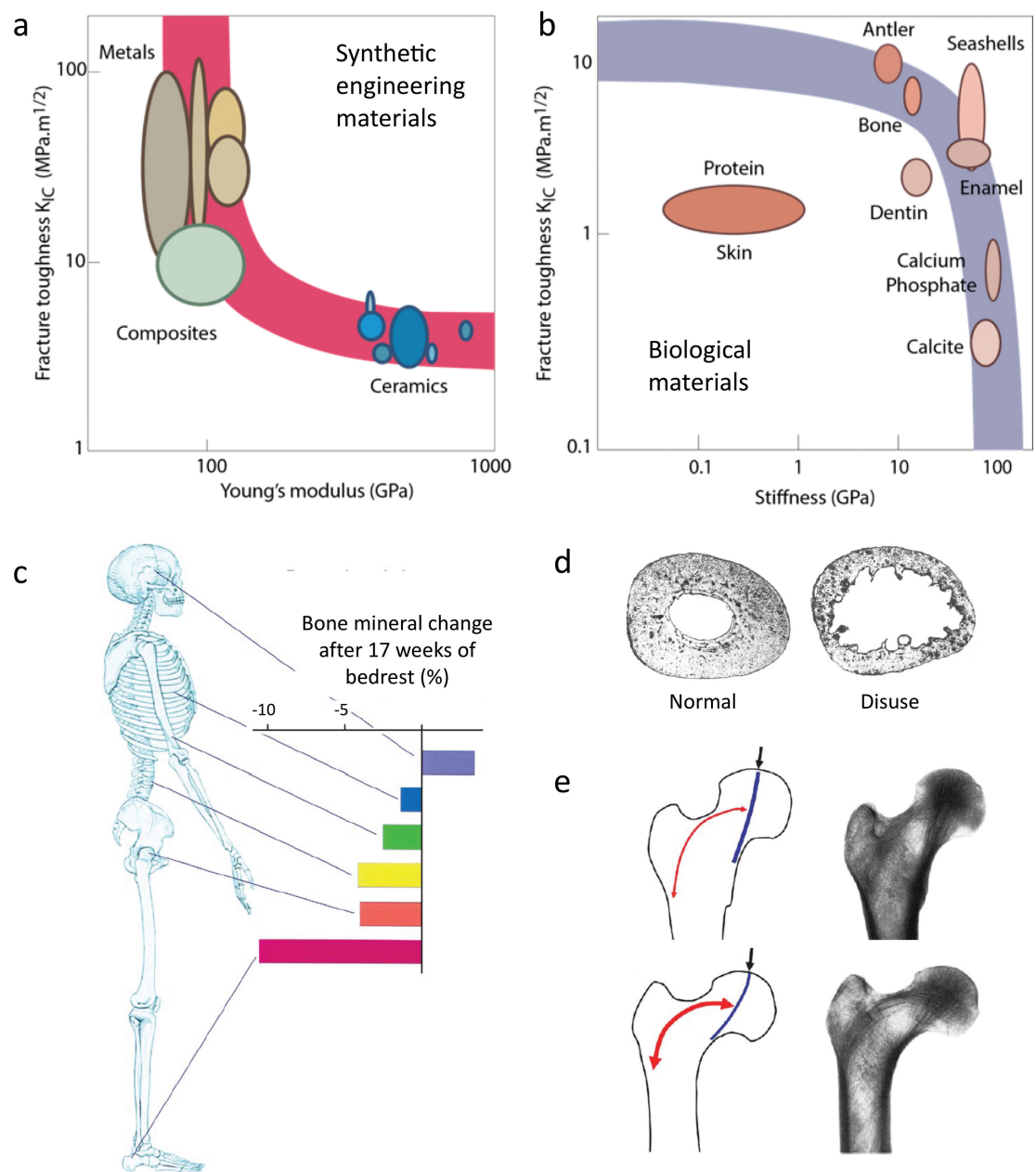

d

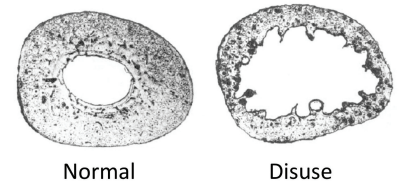

e

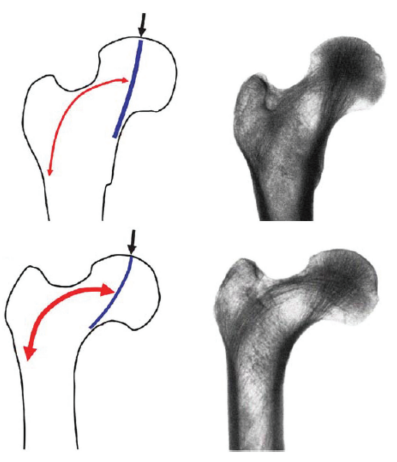

Figure 1: The tough and living nature of human bone. ( $a, b)$ Fracture toughness and stiffness of a wide range of (a) synthetic engineering materials and (b) biological materials. A trade-off between these properties is often found in engineering materials, but is circumvented in materials made by living organisms. (c) Relative change in bone mass in different skeletal parts of the human body after resting in bed for 17 weeks. (d) Cross-sections of the metacarpal bone of an adult dog after 40 days of disuse compared to the normal structure. The metacarpal bone is positioned between the "wrist" and the toes. (e) X-ray images of two distinct femoral heads showing how the architecture of the trabeculae inside the bones (dark colour) follow the principal stress lines arising from mechanical loading. The red arrows and blue lines indicate the direction of tension and compression stresses, respectively. The example on top develops higher tensile stresses compared to compressive stresses. The situation inverts in the example shown at the bottom. Graphs (a) and (b) were adapted by Dr. Florian Bouville from Espinosa et al. [5] Images (c-e) are adapted and reproduced with permission from Robling et al. [3].

illustrated by the loss of bone mass experienced by astronauts in low-gravity conditions. At the other end of the spectrum, tennis players are known to develop thicker bones in the racket-holding arm compared to the other non-dominant arm bone [2]. Lying in bed for several months is sufficient to cause significant variations in bone mass along the human body (Figure 17) [3]. Besides gaining or losing mass, bone is also able to adapt its internal architecture to match the stress lines developed within the tissue during mechanical loading (Figure 11d). Known as Wolff's law, this rule is a prototypical example of how structural materials in biology can dynamically adapt their structure to fit their function as opposed to the static nature of conventional engineering materials [4]. The dynamic and adaptive nature of bone results from the metabolic activity of the living cells that control the biochemistry of this tissue. These comprise primarily bone-forming osteoblasts, bone-dissolving osteoclasts and osteocytes embedded in the extracellular matrix that constitute this mineralized tissue. Together such cells are able to locally grow or dissolve bone in the regions of, respectively, high or low mechanical stresses, and to continuously renew the tissue to remove damage developed in the structure during its lifetime. As a result of these cellular processes, the average turnover of the mineral phase of our bones is as high as $10 \%$ and $20 \%$ every year for cortical and trabecular bone, respectively. The mineral turnover can be maintained at stable levels through the balanced action of osteoblasts and osteoclasts, leading 
to a dynamic equilibrium state known as homeostasis In addition to damage repair, bone homeostasis is important to maintain the level of calcium in the blood stream. The interplay between the large number of osteoblasts, osteoclasts and osteocytes present in bone is at the core of the self-organization processes, hierarchical structures and adaptive response of this complex living material.

In this review article, we discuss the current understanding of the biological material bone from the perspective of complex systems. Starting at the cellular level, the self-organization processes underlying the formation of the collagen phase and its subsequent mineralization with calcium phosphate are discussed. Next, we describe the hierarchical structure that emerges from such self-organization processes and explain how structural features at different length scales of the tissue give rise to the high toughness of bone. Finally, experiments and models that have been proposed to explain bone's unique ability to adapt to its environment are reviewed in light of universal features observed in complex systems. Understanding the properties and dynamic behavior of bone requires basic knowledge from a wide range of disciplines, including physics, chemistry and biology. To help the reader navigate through these distinct disciplines, we have included tutorial panels that focus on the fundamental concepts needed to fully appreciate the complexity of this fascinating material.

\section{Self-organization}

Bone tissue is formed by an exquisite sequence of cellmediated self-organization processes that starts with the formation and assembly of collagen fibrils, and is followed by the mineralization of such fibrils with the inorganic calcium phosphate phase. Below, we describe how collagen fibrils are assembled and mineralized, highlighting the cellular processes and the supramolecular interactions involved.

\subsection{The assembly of collagen fibrils}

Collagens are the most abundant family of proteins present in vertebrates and the main source of tensile strength and elasticity in animal tissues. Its precursor, the procollagen $\alpha$-chain, is synthesized by elongated fibroblasts (Figure 2a) as well as by highly branched and mononucleated cuboid cells called osteoblasts. Procollagens are polypeptide chains comprised of two parts that have different amino acid sequences. The central part exhibits a collagenous domain that is characterized by repeated sequences of amino acids residues in the form of Gly (glycine)-X-Y, with X and Y being typically proline (Pro) and hydroxyproline (Hyp), respectively (orange domain in Figure 2p). As the smallest amino acid, glycine plays an important role in the assembly

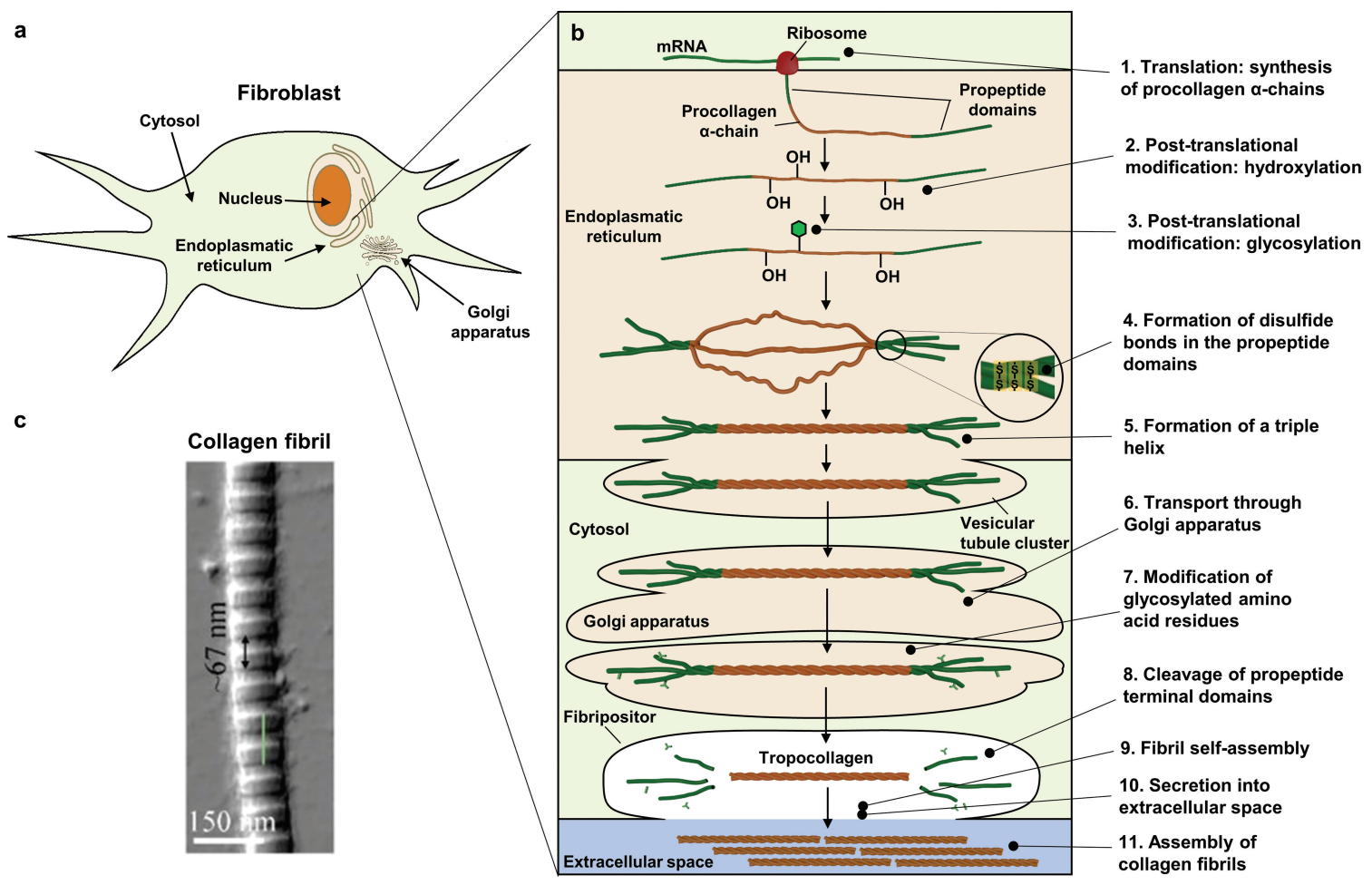

Figure 2: Synthesis and self-organization of collagen fibrils. (a) Fibroblasts synthesize collagen precursors in the endoplasmatic reticulum and secrete them into the extracellular space. (b) Multiple steps involved in the formation of collagen fibrils, including the synthesis and chemical modifications of procollagen chains, their folding into tropocollagen triple helices and self-organization into larger collagen fibrils. (c) Atomic Force Microscopy (AFM) image of a collagen fibril depicting its striated morphology derived from the staggered packing of tropocollagen triple helices. Adapted and reproduced with permission from Andriotis et al. [7]. 
of collagen. The repeated occurrence of this amino acid allows the $\alpha$-chains to fold into highly curved left-handed helices that can intertwine with two other $\alpha$-chains to eventually form right-handed triple-helical protein complexes [6]. In contrast to this central domain, the terminal parts of the $\alpha$-chain consist of amino-terminal domains characterized by a sequence of amino acids that results in globular folding (green domains in Figure 2p). In this section, we will present the current understanding of the synthesis and intracellular chemical transformation of procollagen chains and their transportation, folding and secretion into the extracellular space to form the hierarchically organized fibrillar structures known as collagen fibrils.

Similarly to the production of other protein complexes in eukaryotic cells, collagen molecules are synthesized inside the cell and secreted into the extracellular space following a series of steps that are executed at specific sites within the cell. This process resembles the typical assembly line of a product in a factory [8]. The production of collagen initiates in the cell's nucleus, where the genetic information is decoded and transcripted into mRNA molecules. To carry the coding sequence further, mRNA molecules cross the nuclear membrane and reach the ribosomes that are attached onto the membranes of the endoplasmatic reticulum (ER). Ribosomes then read the coding sequence and start connecting amino acid residues to make procollagen $\alpha$-chains, the synthetic precursors of the family of collagen proteins (Step 1, Figure 2b) 9]. Inside the ER, procollagen $\alpha$ chains are modified by the covalent addition of functional groups to specific amino acid residues that can ultimately stabilize the triple helical conformation (see tutorial box, Figure 3). First, the hydroxylation of proline and lysine residues increases the number of hydrogen bonding sites and enhances the stabilization of the triple helix conformation through non-covalent interactions (Step 2, Figure 2b). Next, some of the hydroxylysine residues are converted into glycans by addition of carbohydrate units through enzymatic glycosylation (Step 3, Figure 2p). Although the function of glycan groups in collagen still remains unknown, alterations of the glycosylation level of collagen have been reported in several bone disorders related to biomineralization, fibrillar crosslinking and collagen remodeling [10 12. Following glycosylation, non-covalent interactions of amino acid residues present in the terminal propeptide domains lead to the formation of the trimeric procollagen unit. This unit is subsequently stabilized by the formation of interchain covalent disulfide bonds through oxidation of cysteine residues (Step 4, Figure 2b) [13]. The covalent association through disulfide bonds likely triggers the zipper-like folding of the procollagen domains into the triple helix conformation (Step 5, Figure 2b). Intraand interstrand supramolecular interactions within the procollagen domains are crucial for the stabilization of the triple helix conformation (Figure 3.
As the collagenous protein complexes are much larger $(300 \mathrm{~nm})$ than the transportation vesicles $(60-80 \mathrm{~nm})$ typically produced by cells, special membrane-bound compartments called vesicular tubular clusters are assembled to transport the collagen complex from the ER to the Golgi apparatus (Step 6, Figure 2b). Once the complex molecule enters the Golgi apparatus, the glycosylated residues of the propeptide domain are further modified with chains containing multiple carbohydrates units (Step 7, Figure 2b). Chemical modifications mediated by enzymes present in the Golgi apparatus seem to act as a signal that instruct the cell's transportation system to deliver the procollagen molecules to their final destination within the cell [14]. This final intracellular transportation process is carried out by membranebound compartments known as fibripositors. Next to the plasma membrane, enzymatic cleavage of the terminal propeptides chains results in the formation of tropocollagen triple helices (Step 8, Figure 2p) that eventually self-organize into collagen fibrils (fibrillogenesis, Step 9, Figure $2 \mathrm{p}$ ). Finally, the membranes of the fibripositor and the cell fuse to secrete the initial fragments of collagen fibrils into the extracellular space (Step 10, Figure 2b). Fibrillogenesis continues in the extracellular space (Step 11, Figure 2p) where each tropocollagen triple helix is offset along the longitudinal axis by a distance (D) of $67 \mathrm{~nm}$, to yield a periodic crossstriated structure with well-defined gap (0.6D) and overlap (0.4D) zones within the assembled collagen fibril (Figure 3). The fibrils show average diameter ranging typically from 10 to $500 \mathrm{~nm}$ and length that can reach up to a few millimeters in size (Figure 2k). The mechanical strength and stiffness of such fibrils are afterwards enhanced through a biomineralization process.

\subsection{Biomineralization}

Biomineralization in bone tissues is still a topic of debate in the scientific community. This process is regulated by osteoblasts (Figure 4a) and occurs after the non-mineralized collagen matrix is deposited on the bone surface as osteoid seams. In healthy bones, the time between osteoid formation and the onset of the mineralization process, known as mineralization lag time, typically ranges between 2 and 3 weeks [21. In the last decades, several models have been proposed to explain the mineralization of collagen fibrils in bone through the formation of the crystalline calcium phosphate phase hydroxyapatite. Some of these models include (i) cell-independent processes, in which noncollagenous proteins interact with the gap zones in collagen fibrils, activate the capture of ions and trigger the crystallization of hydroxyapatite [22]; (ii) cellcontrolled processes, in which vesicles that are secreted by mineralizing cells accumulate ions in the extracellular space, deliver their contents to the collagen fibrils, and induce hydroxyapatite precipitation [23]; and (iii) 


\section{The supramolecular chemistry of collagen}

Structural fibrils in bone and in other connective tissues are formed through the supramolecular assembly of the protein collagen (type I). Proteins are predominantly linear macromolecules with chains comprised of a programmed sequence of molecular building blocks called amino acids. Each amino acid consists of a central carbon atom ( $\alpha$-carbon) that forms chemical bonds to an amino group, a carboxylic acid group, a hydrogen atom and a specific side group, $R$ (Figure 3.1a). Most of the naturally occurring proteins are formed by the combination of different amino acids that are connected to each other through peptide bonds. The specific sequence of amino acids along a protein chain is defined by the information encoded in the genetic code of the organism.

When exposed to water, proteins self-assemble into a myriad of complex structures that ultimately confers their functionality. This self-assembly process results from the development of intra- and intermolecular non-covalent interactions of high specificity and affinity. During folding in water, the more hydrophobic side groups along the protein must be shielded to reduce the free energy of the system. This is partially achieved by the formation of hydrogen bonds between the more polar groups of the main chain, namely the carbonyl oxygen and the amide hydrogen of the peptide bond (Figure 3.1b). Supramolecular chemistry explains how these non-covalent interactions enable the formation of highly complex systems that exhibit functionalities going beyond the simple chemical structure of discrete molecules.

$$
\text { a }
$$

Amino acid

Peptide bond

$$
\text { Hydrogen bonds }
$$

Figure 3.1. (a) Chemical structure of amino acids and the formation of a peptide bond from the condensation reaction of two amino acids. (b) Linear protein chains depicting hydrogen bonds formed between the polar groups of the main chain.

For the assembly of collagen fibrils, programmed supramolecular interactions between tropocollagen molecules lead to the formation of a triple helical fold (Figure 3.2a). Such folds are characterized by long amino acids sequences comprised of Glycine (Gly) Proline (Pro), and 4R-hydroxyproline (Hyp) (Figure3.2b). Protein chains containing sequences of these amino acids assemble into stable right-handed super helices that are held together by a network of cooperative hydrogen bonds formed between the hydrogen amide of the Gly in one strand and the carbonyl oxygen of the Pro or Hyp of an adjacent strand. Additional stability of the triple helix conformation is achieved through the formation of salt bridges between ionizable side groups of the amino acids in the main chains. X-ray diffraction and Nuclear Resonance (NMR) studies employing minimalist models of similar ionizable amino acid sequences show the formation of intra- and interstrand salt bridges that help minimize the free energy of the folded system (Figure 3.2c).

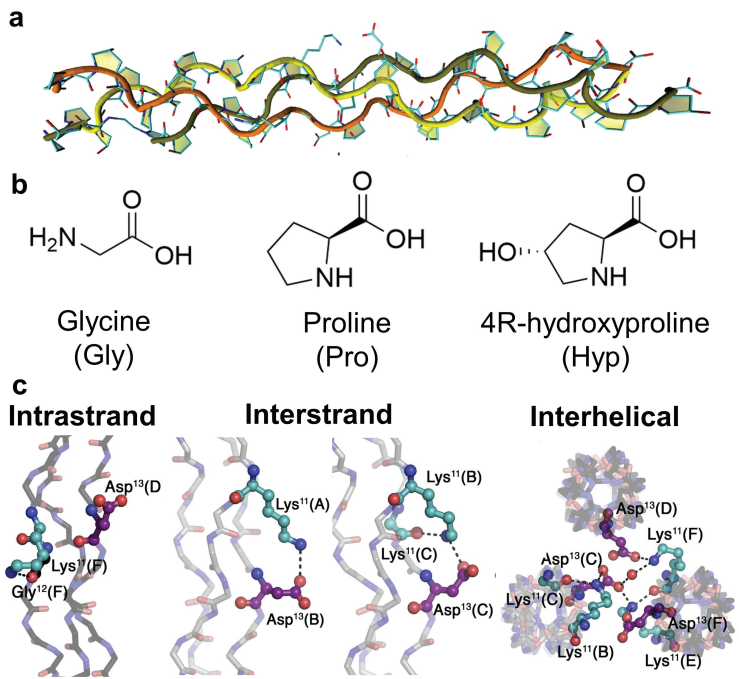

Figure 3.2. (a) Three-dimensional model of the tropocollagen triple helix. (b) The three amino acids involved in the main supramolecular interactions leading to the triple helical fold. (c) Additional supramolecular interactions that contribute to the stabilization of the triple helix and the quasihexagonal packing of helices.

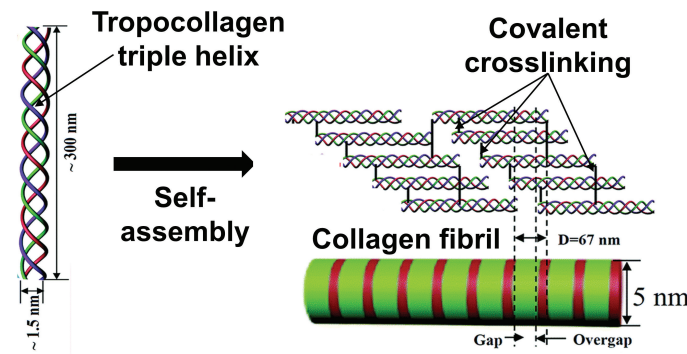

Figure 3.3. Self-assembly process of tropocollagen triple helices into collagen fibrils highlighting the formation of covalent crosslinking between adjacent helices.

To form a collagen fibril, tropocollagen triple helices self-assemble into periodic cross-striated micrometer-sized structures that exhibit a staggered lattice (Figure 3.3) with a quasi-hexagonal cross-section packing. This selfassembly process is driven by the interstrand and interhelical supramolecular interactions that arise from ionizable amino acid side groups (Figure 3.2c). In addition to these supramolecular interactions, tropocollagen triple helices are also connected via covalent crosslinks within the collagen fibril (Figure 3.3). This covalent crosslinking provides additional mechanical and thermal stabilities to the collagen fibrils.

Figure 3: Tutorial on the supramolecular chemistry of collagen fibrils [15-19]. All images except 3.2c are adapted and reproduced with permission from Zhu et al. and Hahn [19] 20]. Image 3.2.c was originally published in the Journal of Biological Chemistry [18] (C. 

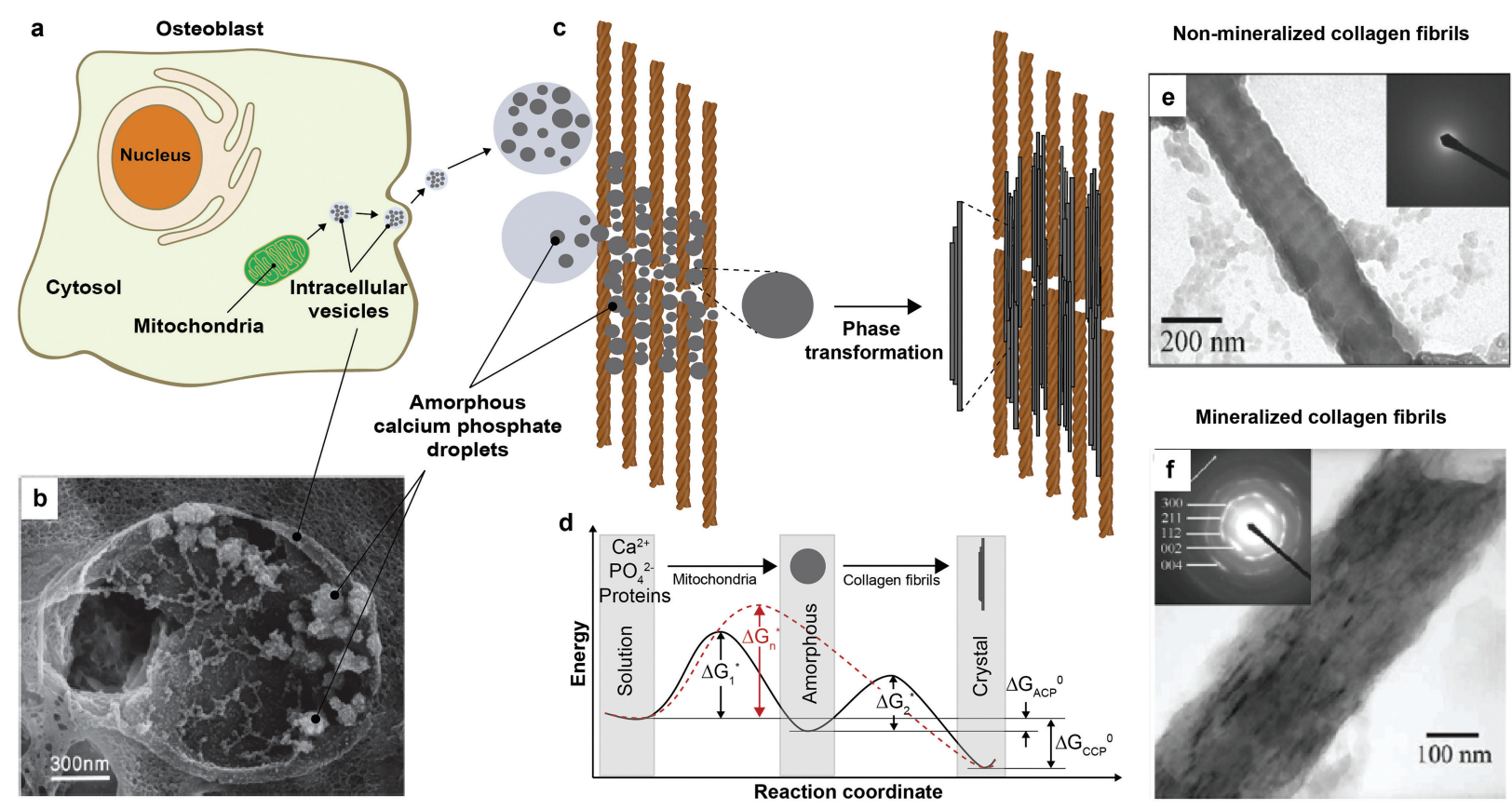

Figure 4: Mechanism proposed for the biomineralization of bone tissue. (a) Osteoblasts produce intracellular vesicles loaded with amorphous calcium phosphate droplets and secrete them into the extracellular space. (b) Cryo-Scanning Electron Microscopy (SEM) image depicting an intracellular vesicle with multiple amorphous calcium phosphate droplets. Reproduced with permission from Addadi and Weiner [25]. (c) Cell-controlled model for the biomineralization of collagen fibrils, highlighting the phase transformation from an amorphous precursor to oriented platelet-like crystals. (d) Energy landscape for the crystallization process, comparing the activation energies involved in the classical nucleation and growth theory ( $\Delta G_{n}^{*}$, dashed red line) and the phase transformation model $\left(\Delta G_{1}^{*}\right.$ and $\Delta G_{2}^{*}$, continuous black line). (e) Transmission Electron Microscopy (TEM) image of a non-mineralized collagen fibril. The electron diffraction image shown in the inset indicates the absence of crystalline minerals in the collagen fibril. (f) TEM image of a mineralized collagen fibril. The electron diffraction displayed as inset shows diffraction peaks assigned to hydroxyapatite crystals that have their c-axis oriented parallel to the direction of the fibril. Images (e) and (f) are adapted and reproduced with permission from Nudelman et al. [26].

cell-controlled processes, in which amorphous calcium phosphate is transported via intracellular vesicles and later undergoes phase transformation into hydroxyapatite particles within the collagen fibrils [24]. Recently, strong experimental evidences have been shown to support the phase transformation model. According to this model, intracellular vesicles containing amorphous droplets of calcium-based mineral precursor are produced within the mitochondria of osteoblasts (Figure 4 a). In this section, we will discuss the experimental observations that support this biomineralization model of bone tissue.

Mitochondria are membrane-bound organelles responsible for the storage of chemical energy in the form of adenosine triphosphate (ATP) molecules inside eukaryotic cells. In these organelles, calcium ions are internally accumulated to balance the electric potential that is created by the proton gradient used to power the enzyme ATP-synthase [27]. Such calcium ions form insoluble complexes in the presence of proteins and phosphate ions, which are released into the cytosol as droplets inside membrane-bound vesicles (Figures 4 a , b) $[2832$. These intracellular vesicles are transported through the cytosol, secreted into the extracellular space and end their journey by delivering their content onto non-mineralized collagen fibrils located at the mineralization front of the bone tissue (Figure 4, e). Once released, the amorphous calcium phosphate from the droplets binds to specific sites located at the end of the tropocollagen triple helices, diffuses and solidifies within the gap zones of the collagen fibrils [33, 34]. Eventually, the amorphous precursor undergoes a phase transformation into oriented platelet-like hydroxyapatite crystals (Figure 4, f).

Although classical nucleation theory has been used to explain the crystallization of hydroxyapatite particles in bone tissue 35, the phase transformation model described above is in good agreement with several experimental observations [24, 30, 31, 36]. Biomineralization via phase transformation of an intermediate amorphous phase reduces the flow of water during crystallization, enables pre-shaping of the amorphous phase into defined and unusual geometries, eases transportation of precursors to their final destinations, and enhances the crystallization rates up to two orders of magnitude compared to classical nucleation and growth mechanisms [37, 38]. The remarkably faster crystallization achieved by phase transformation results from the reduced activation energy for the formation of the metastable amorphous 
phase $\left(\Delta G_{1}^{*}\right.$, continuous black line in Figure $\left.4 \mathrm{~d}\right)$ in comparison to the energy barrier $\left(\Delta G_{n}^{*}\right.$, dashed red line in Figure $4 \mathrm{~d}$ ) involved in the nucleation and growth of ion clusters in the classical mechanism [34]. This suggests that the biomineralization process occurs via pathways that facilitate the crystallization of reinforcing mineral platelets within the collagen fibrils.

Besides their prominent role in the assembly of collagen fibrils (Figure 3), non-covalent molecular interactions have also been proposed to explain the controlled crystallization of platelet-like hydroxyapatite particles in bone tissues. Although the current spatial and temporal resolutions of in vitro and in vivo experiments are not sufficient to fully elucidate the interplay between the supramolecular assembly of collagen and the phase transformation of the mineral phase [39], functional groups present in the tropocollagen triple helix seem to work in synergy with inhibitors of hydroxyapatite nucleation so as to actively control the process of mineralization. Additionally, charged amino acids located at the end of the tropocollagen triple helices may attract electrostatically the amorphous calcium phosphate droplets and favor their infiltration into the collagen fibrils. These charged amino acids residues can also serve as heterogeneous nucleation sites to induce the phase transformation of amorphous calcium phosphate precursor into parallel arrays of oriented hydroxyapatite crystals [40. The elaborate biological and chemical processes behind the synthesis of mineralized collagen fibrils well illustrate the complex nature of the bone formation process at its smallest length scales. Beyond the scale of individual fibrils, bone features a sophisticated hierarchical architecture that finds no counterparts among structural engineering materials.

\section{Hierarchical Structure}

\subsection{Structural features across length scales}

The assembly of collagen molecules and hydroxyapatite nanocrystals into mineralized fibrils is the result of a self-organization process driven by individual bone cells. The collective action of such cells eventually gives rise to an exquisite hierarchical architecture that spans from the nano- to the centimeter scale and makes bone a lightweight material with unusually high stiffness and fracture toughness (Figure 5a) [41]. To understand how these mechanical properties emerge and to appreciate the complexity of this biological material, we describe the main structural features of bone from the largest to progressively smaller length scales.

At the macroscopic scale $\left(\sim 10^{0} \mathrm{~cm}\right)$, bone displays a core-shell cylindrical morphology comprising a dense outer shell known as cortical bone and a highly porous inner core referred to as trabecular bone 42. Trabecular bone contains about $20 \mathrm{vol} \%$ of mineralized tissue, the rest being occupied by marrow and fat. The mineralized tissue in the core forms a load-bearing continuous open network that provides shock resistance and transfers mechanical loads to cortical bone. Following a mechanical design often used in engineering structures subjected to bending, the low fraction of mineralized tissue in trabecular bone enables weight reduction without compromising the mechanical performance of the overall structure. Indeed, $80 \%$ of the overall bone mass is present in cortical bone, where the highest mechanical stresses are applied [42].

To cope with the high stresses developed during running and jumping, cortical bone is organized in a hierarchical architecture that displays high strength and fracture resistance. At the scale of hundreds of micrometers, the main structural elements of cortical bone are the osteons. These are concentric lamellar structures featuring a central channel that hosts blood vessels and nerves, which is known as the Haversian canal 41. In cortical bone, the osteons reinforce bone in a similar way to fibers in engineering composite materials. Efficient reinforcement is achieved due to the orientation of the osteons along the direction of the highest mechanical stresses within cortical bone. The fiber-like osteons are embedded in the extraosteonal bone matrix and surrounded by 1 to $5 \mu \mathrm{m}$-thick cement lines 44, 45. Because of their softer nature and low mineral content, cement lines play a key role in the high fracture resistance of bone, as discussed later in the text [46.

At smaller length scales, each osteon is composed of concentric lamellae that are reinforced by arrays of mineralized collagen fibrils. The orientation of individual collagen fibrils varies within each osteonal lamella, leading to sublayers of fibrils aligned in specific directions [43, 47]. Several models have been proposed to describe the fibrillar alignment patterns across the different sublayers in the osteonal lamellar tissue [48 51]. The recent identification of twisted and oscillating arrangements within the same osteon suggests that the fibrils follow complex and mixed orientation patterns in the osteonal lamellar tissue. With a diameter of $\sim 100 \mathrm{~nm}$ and length up to $\sim 10 \mu \mathrm{m}$, a fibril is selfassembled from collagen molecules of approximately $1.5 \mathrm{~nm}$ thickness and $300 \mathrm{~nm}$ length. In the selfassembly process, hydroxyapatite nanoplatelets grow in the gaps between the collagen molecules, as explained in the previous section. At the nanoscale, the collagen fibrils are composed of staggered arrays of tropocollagen triple helices that are held together by supramolecular interactions [52].

Our current understanding of the hierarchical architecture of bone has relied on characterization techniques that enable the analysis of structural features spanning across multiple length scales. Synchrotron-based X-ray diffraction methods (Wide-Angle X-ray Diffraction and Small-Angle X-ray Scattering), electron microscopy (Scanning and Transmission Electron Microscopy) and vibrational spectroscopy approaches are some of the 
a

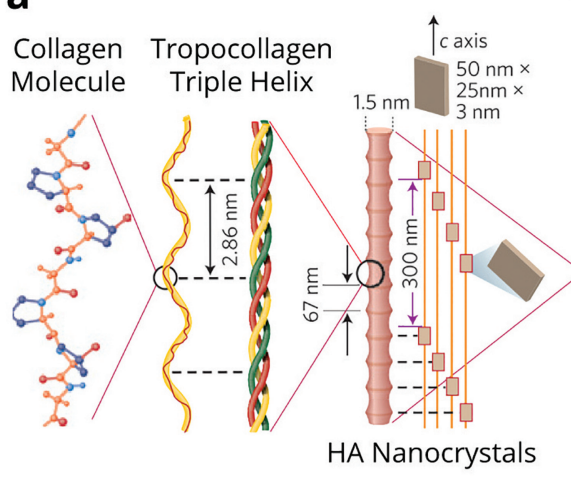

b Osteonal Tissue

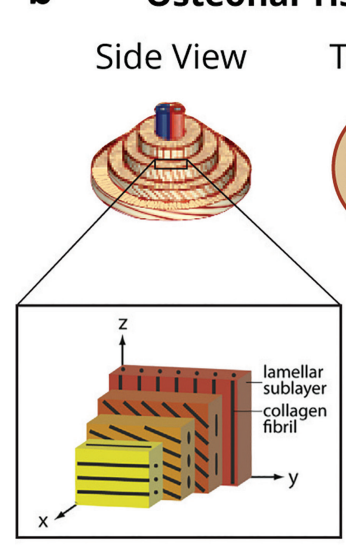

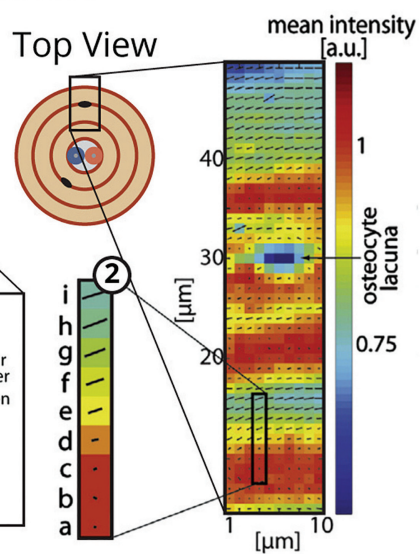

Collagen Fibril
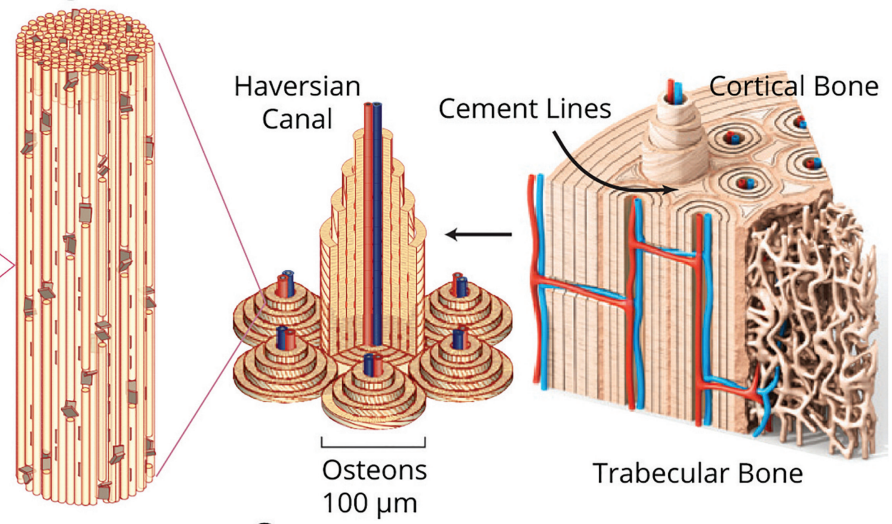

C

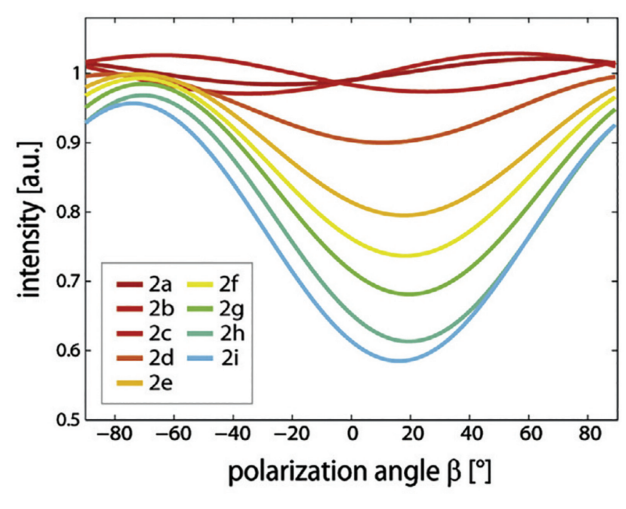

Figure 5: The hierarchical structure of bone. (a) Schematics of the hierarchical architecture of bone, highlighting the main structural features of this biological tissue across multiple length scales. Adapted and reproduced with permission from Wegst et al. [41]. (b) Orientation of collagen fibrils within the cross-section of an osteon obtained from polarized Raman spectroscopy of bone tissue. The color code represents the average intensity of the amide I Raman band arising from the collagen molecules. (c) Intensity of the amide I Raman band as a function of the polarization angle of the incoming laser for different positions in the osteonal cross section. Adapted and reproduced with permission from Schrof et al. [43].

techniques that have been utilized for this purpose [53]. To illustrate how these tools can shed light on the intricate architecture of biological materials, we briefly describe the use of polarized Raman spectroscopy for the analysis of the orientation of collagen fibrils within the lamellae and sub-lamellae of osteons (Figure 5b) 43.

Polarized Raman spectroscopy is a non-destructive characterization method that has been applied to obtain structural and chemical information about the collagen and mineral phases of bone. The technique relies on the inelastic scattering of light resulting from interactions with the probed material. Such scattering leads to a frequency shift in the Raman signal that can be assigned to a vibrational mode of a specific chemical group. Since the intensity of Raman scattering is influenced by the orientation of the incident polarized light relative to the vibrating molecular units, this method provides an effective means to quantify molecular alignment in anisotropic materials. In the case of bone, the amide groups of the collagen molecules generate an anisotropic Raman active vibrational band $\left(\sim 1660 \mathrm{~cm}^{-1}\right)$ that has been used to map the orientation of the mineralized collagen fibrils across a cross section of an osteon. The gradually changing orientation of mineralized fibrils within an osteon lamella is evident from the strong dependence of the Raman intensity signal on the polarization of the incident laser (Figure 55) [43, 54]. Orientation maps obtained with this analysis reveal that the concentric lamellae within the osteon is composed of sublayers displaying collagen fibrils aligned in a specific direction. As the orientation of the collagen fibrils changes between distinct sublayers, this analysis confirmed the coexistence of twisted and oscillating plywood patterns in the fibrillar alignment within the same osteonal lamellar tissue [43]. Such a plywood-like arrangement of fibrils is expected to improve the resistance of bone against fracture by enabling the arrest and twisting of cracks at the length scale of the osteon lamellae.

\subsection{Strength and fracture toughness}

The high fracture resistance of cortical bone arises from reinforcing and toughening mechanisms operating at multiple length scales. As in engineering materials, 


\section{Cooperative deformation}

a

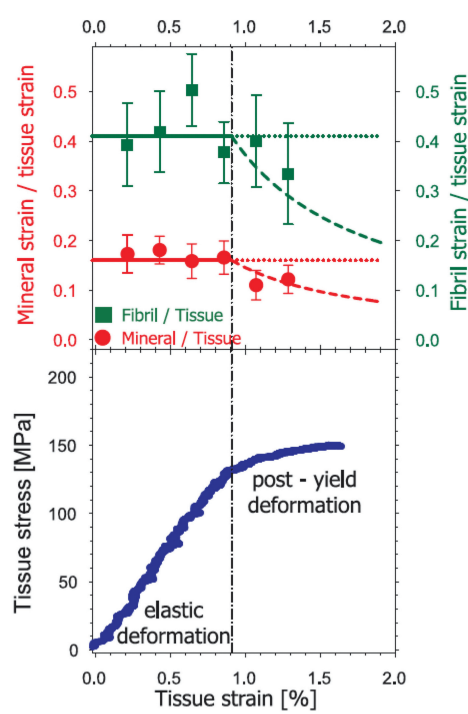

b

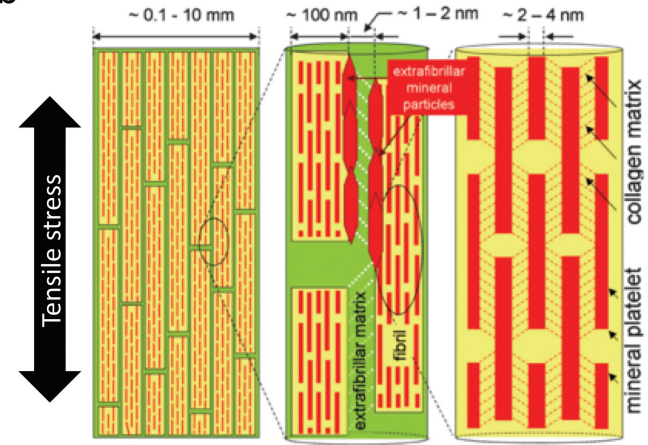

Crack deflection and twisting

C

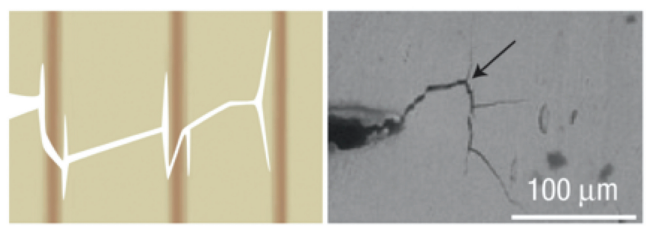

d

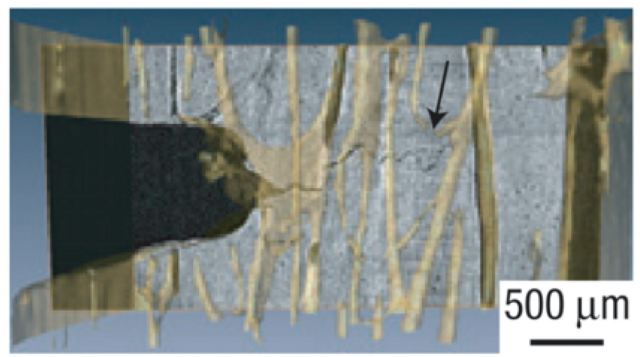

e

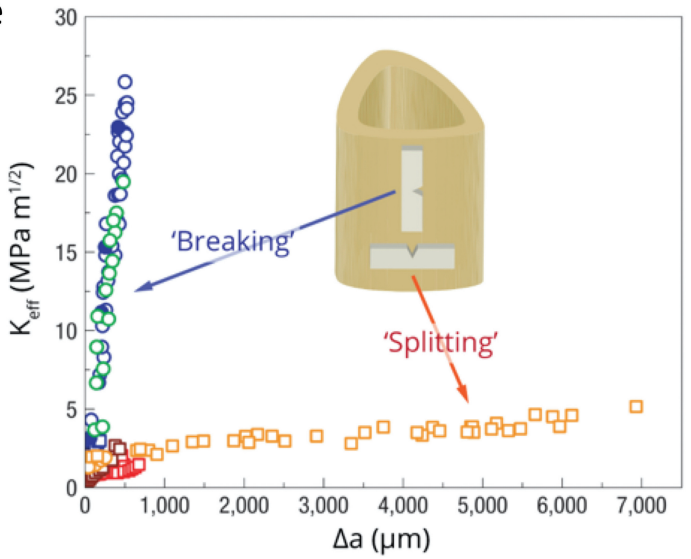

Figure 6: Strengthening and toughening mechanisms in cortical bone. (a) Measurements of the relative strain in the mineral platelets and in the mineralized collagen fibrils during tensile testing of a sample of bone tissue along the longitudinal direction. The relatively high strain detected in these structural elements demonstrate the importance of cooperative deformation mechanisms for the strength and stiffness of bone. (b) Schematic model depicting the transfer of stress to stiffer elements via shear stresses developed within the matrices (dashed lines) at multiple length scales. (c) Sketch and scanning electron microscopy image depicting the propagation of a crack along the transverse direction within a bone specimen (perpendicular to the orientation of osteons). In this orientation, strong crack deflection is observed at the cement lines around the osteons. (d) 3D tomographic reconstruction of a bone specimen during transverse fracture, highlighting twisting events that occur when the main crack hits osteons. (e) Resistance of cortical bone against crack propagation in the transverse (breaking) and longitudinal (splitting) directions as a function of crack extension (R-curves). $K_{I R}$ represents the critical stress intensity factor required for crack growth (Figure 7). Figures a and b were adapted and reproduced with permission from Gupta et al. [55]. Figures $\mathrm{c}$ and $\mathrm{d}$ were adapted and reproduced with permission from Koester et al. [57].

fracture in bone is dictated by the interactions of a propagating crack with the structure of the material. While several structural features may provide resistance against crack propagation in a material [55, 56] we focus on two important reinforcing and toughening mechanisms that contribute to the high strength and fracture toughness of cortical bone (Figure 6a), namely (1) multiscale cooperative deformation and (2) crack deflection and twisting. By operating in a complementary fashion at multiple length scales, these mechanisms highlight the importance of the hierarchical structure for the mechanical properties of bone. A more detailed analysis of these toughening mechanisms is essential to understand how bone can exceed the mechanical properties expected from its relatively soft collagen and weak hydroxyapatite building blocks.

The cooperative deformation of fibril arrays, individual mineralized collagen fibrils and mineral platelets at progressively smaller length scales has been suggested to be an important contribution for bone's high strength [55]. This has been demonstrated by performing in situ synchrotron-based X-ray scattering measurements on bone samples subjected to external mechanical forces (Figure 6a). The central questions in such experiment were: how are the applied external forces and strains distributed inside such a material 
containing stiff and soft phases at different length scales? Which of the structural elements present at multiple length scales in bone contributes to the global stiffness and strength of the material? To answer these questions, the strain developed within the mechanically loaded material at increasing length scales was experimentally measured. Wide-Angle X-ray Diffraction (WAXD) was used to quantify the strain at the scale of the mineral platelets, whereas the strain at the coarser level of mineralized collagen fibrils was measured by SmallAngle X-ray Scattering (SAXS). The diffraction and scattering data were complemented by the global strain at the tissue level measured directly on the macroscopic sample.

The in situ synchrotron measurements on bone samples show that both mineralized collagen fibrils and mineral platelets are strained upon the application of increasing external tensile forces. When normalized with respect to the tissue strain, the strain level on these two structural elements was found to remain constant during the initial elastic deformation of the tissue, before decreasing monotonically once the material starts to deform plastically (yielding). These results support the idea that global stresses are transmitted throughout the hierarchical structure of bone by shear forces in the extrafibrillar matrix that are translated in tensile forces in the mineralized collagen fibrils, which in turn develop shear forces in the collagen matrix that are converted into tensile stresses in the mineral platelets (Figure 60). This coordinated stress transfer mechanism across hierarchical levels makes it possible for all the structural elements to contribute to the overall stiffness of bone, while maintaining the local strain levels below the critical limit that would cause failure [55].

While cooperative deformation is an important mechanism to enhance bone's stiffness and strength, the resistance of bone against fracture arises mostly from the deflection and twisting of cracks at the cement line between osteons 41. Cracks propagating in the transverse direction perpendicular to the orientation of the osteon are strongly deflected at the cement line due to the weak nature of this interfacial layer. Such deflection moves the crack tip away from the plane of maximum tensile stress, strongly reducing local stress concentration and thus requiring higher loads to propagate the fracture further [57]. Because the cement lines form a weak interface also along the circumferential direction around the osteons, in-plane crack deflection is accompanied by out-of-plane twisting of the crack. This twisting effect reduces further the stress concentration at the crack tip and leads to significant toughening [57. The deflection and twisting of transverse cracks at cement lines of cortical bone undergoing fracture have been confirmed by scanning electron microscopy and in situ synchrotron X-ray computed tomography (Figure 6e, d) [57.

The toughening effect induced by crack deflection and twisting can be quantified by measuring the resistance imposed by the material against crack propagation as the crack grows larger into the microstructure. The resistance of the material against crack growth is often expressed in terms of the critical stress intensity factor at the crack tip, $K_{I R}$ (see tutorial panel, Figure 7). Measurements of $K_{I R}$ as a function of crack extension, known as the R-curve, are used to quantify the evolution of the fracture toughness of the material during crack propagation. Experimental R-curves show that the fracture toughness of cortical bone increases by a factor of five or more as a crack penetrates $0.5 \mathrm{~mm}$ in the transverse direction (Figure 6e). The maximum $K_{I R}$ value of $25 \mathrm{MPa} \cdot \mathrm{m}^{1 / 2}$ achieved rivals that of the toughest engineering ceramics, despite the soft and weak nature of the collagen and hydroxyapatite building blocks. For sake of comparison, the strong glass cover of a smart phone displays fracture toughness lower than $1 \mathrm{MPa} \cdot \mathrm{m}^{1 / 2}$ and no crack growth resistance (rising R-curve), whereas tough zirconia ceramics used in dental applications show a relatively low $K_{I R}$ of approximately $6 \mathrm{MPa} \cdot \mathrm{m}^{1 / 2}$. It is important to note that the presence of large heterogeneities lowers bone's fracture strength compared to these other structural materials (Figure 7) and that the highly anisotropic architecture of bone makes its fracture toughness very dependent on the crack propagation direction relative to the orientation of osteons. Indeed, the $K_{I R}$ value measured for cracks propagating in the longitudinal direction show a relatively minor increase with crack extension, reaching levels five times lower than those achieved in the transverse configuration. This explains why it is easier to split than to break bone. Since transverse cracks are susceptible to the highest stress concentration under the bending loads developed in physiological conditions, the orientation of osteons in bone is designed to provide high toughness in this particular crack propagation direction. Such a match between loading conditions and material architecture illustrates how the skeletal tissue is structurally designed and adapted to best fulfill its biological function.

\section{Adaptation}

The self-organization processes underlying bone formation and the hierarchical architecture responsible for its high fracture toughness strongly rely on the metabolic activity and concerted action of the living cells present in this biological material. Such cells are the key drivers of the modeling and remodeling processes that take place in the skeletal tissue.

Bone modeling typically involves the deposition of new tissue in the periosteal (external) surface of the bone to increase the global rigidity of the structure and the removal of tissue from under-stressed regions in the internal part of bone to minimize weight. This modeling process occurs predominantly during growth and development of the skeleton. In remodeling processes, bone cells work together to replace potentially damaged 


\section{The mechanics of fracture}

A material fractures when the externally applied stress is sufficient to break the atomic bonds that hold the material together. Assuming that the mechanical energy applied to break an atomic bond is totally transformed into surface energy upon fracture, one can show that the theoretical cohesive stress $\left(\sigma_{c}\right)$ of a material can be estimated using the following relation:

$$
\sigma_{c}=\sqrt{E \gamma_{s} / x_{0}}
$$

where $E$ is the Young's modulus of the material, $\gamma_{s}$ is the surface energy and $x_{0}$ is the equilibrium spacing between the atoms.

This theoretical prediction for the strength of a material is however seldom confirmed by experiments. The strength of ordinary window glass, for example, is predicted to be 4 orders of magnitude higher than what is measured in a mechanical test. The explanation for this disagreement dates back to the 1910s, when Inglis demonstrated that elliptical flaws lead to local stress concentration in a mechanically loaded material. Indeed, flaws and cracks prevent a fraction of the material's atomic bonds from contributing to the the cohesive stress. This leads to a monotonic increase in the actual local stress as one approaches the tip of a crack (Figure 7.1). For a linear elastic material subjected to plane stress, the dependence of the local stress $\left(\sigma_{l}\right)$ on the distance $(r)$ from an infinitesimally sharp crack is given by:

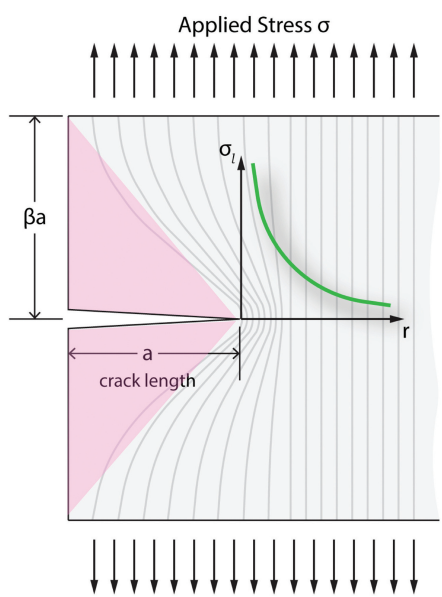

Figure 7.1: Stress concentration near the crack tip in a linear elastic material.

$$
\sigma_{l}=\frac{K_{I}}{\sqrt{2 \pi r}}
$$

where $K_{I}$ is the stress intensity factor under tensile loading (mode I). This factor increases with the crack size (a) and the externally applied stress $(\sigma)$ as follows: $K_{I}=Y \sigma \sqrt{a}$, with $Y$ being a geometrical factor that depends on the crack shape.

While the stress concentration concept effectively captures the effect of flaws on the local amplification of an externally applied stress, the divergence of the local stress as the distance to the crack tip is decreased $\left(\sigma_{l} \rightarrow \infty\right.$ when $\left.r \rightarrow 0\right)$ is not physically meaningful and thus prevents the quantification of the fracture strength of the material.

To solve this dilemma, Griffith put forward an energetic criterion to define the critical stress at which the crack is expected to propagate through the material. He argued that the strain energy initially stored in the atomic bonds above and below a sharp transverse crack (pink area in Figure 7.1) is transformed during fracture into the surface energy of the newly generated crack. Following this argument one can show that the fracture strength of an elastic material in the absence of plastic deformation $\left(\sigma_{f}\right)$ is given by:

$$
\sigma_{f}=\sqrt{\frac{2 E \gamma_{s}}{\pi a}}
$$

This prediction assumes that the strength is determined by an infinitesimally sharp through-thickness crack. Other crack geometries lead to slight variations of the Griffith's equation. Later extensions of this fundamental equation to account for additional energy dissipation mechanisms beyond the formation of new surfaces have led to the more generalized form: $\sigma_{f}=\sqrt{\frac{2 E G_{I C}}{\pi a}}$, where $G_{I C}$ is defined as the critical strain energy release rate and expresses the ability of the material to resist crack growth. Alternatively, the fracture resistance of a material can also be quantified by the critical stress intensity factor $K_{I C}$, which is directly related to $G_{I C}$ through the relation: $K_{I C}=$ $\sqrt{E G_{I C}}$.

Because of its dependence on the size of existing flaws, the fracture strength of brittle (non-plastic) materials is strongly affected by manufacturing processes and therefore cannot be taken as a material property. Instead, the toughness parameters $K_{I C}$ and $G_{I C}$ are properties that better represents the ability of material to resist fracture. Remarkably, tough materials are able to enhance their resistance against fracture as the crack propagates further and interactions with microstructural features, such as grains, fibers and pores. This is often manifested by an increase in the critical stress intensity factor $K_{I C}$ as a function of the extension of the crack $(\Delta a)$, which is referred to as a rising R-curve behavior (Figure 7.2).

a

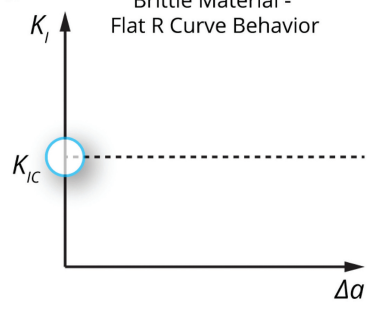

b

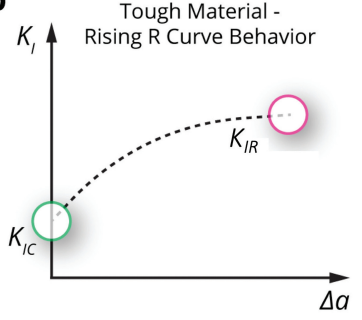

Figure 7.2: Crack resistance curves (R-curves) for (a) brittle materials like ceramics and glasses, and (b) tough materials such as bone, mother-ofpearl and engineering steels. $K_{I C}$ and $K_{I R}$ represent the resistance of the materials against crack initiation and propagation, respectively.

Figure 7: Tutorial on the fracture mechanics of materials [58]. 
a

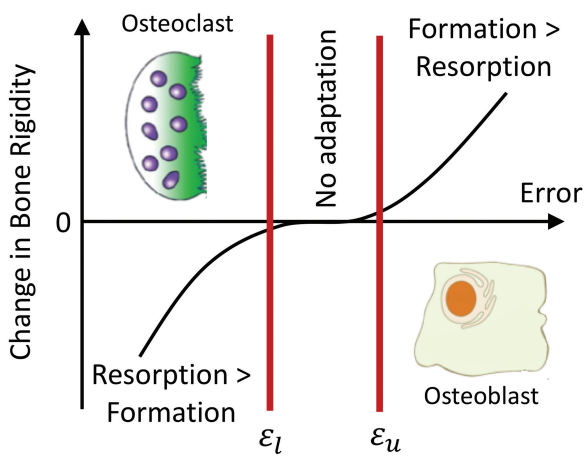

C

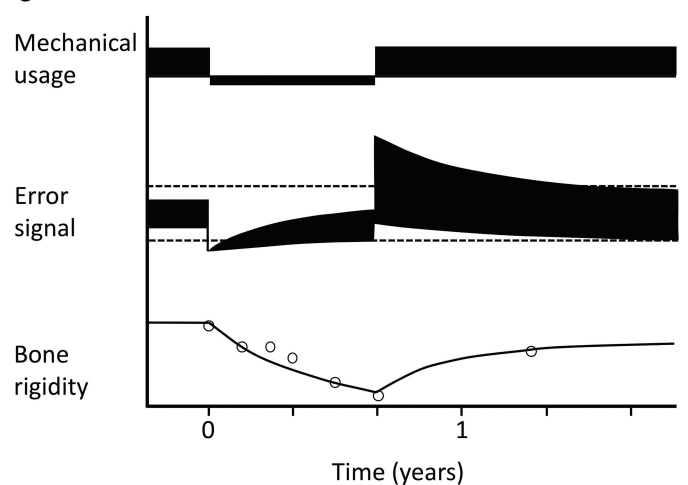

b
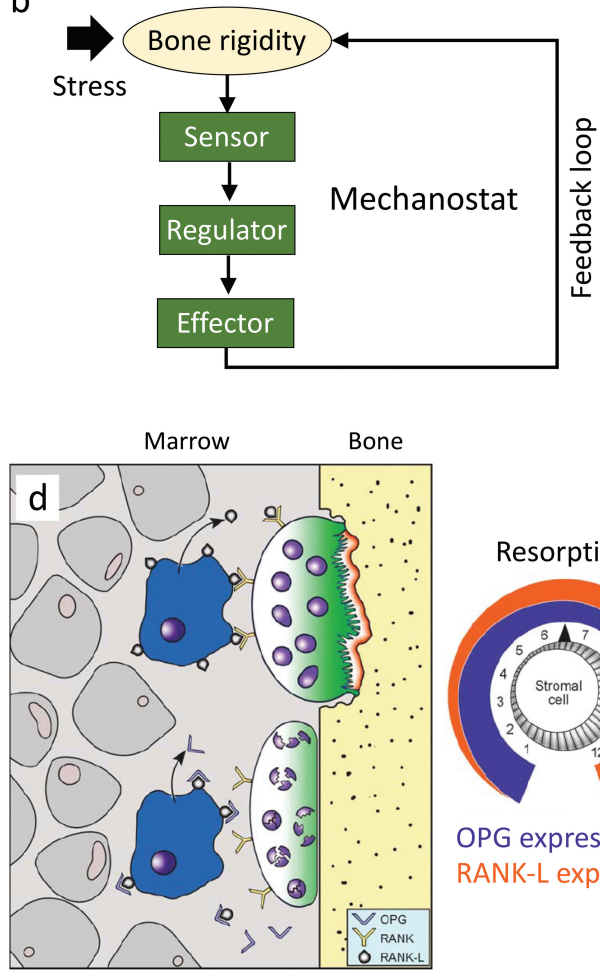

Resorption

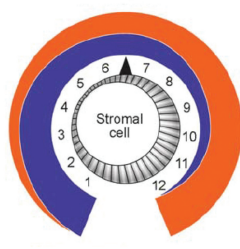

OPG expression RANK-L expression

Figure 8: Bone adaptation modeled as a mechanostat. (a) Change in bone rigidity expected from adaptation depending on the local strain level developed within the tissue [59]. (b) Negative feedback closed loop used to describe the adaptive behavior of bone [59]. (c) Predictions of the mechanostat model and comparison with experimental data for bone under disuse followed by remobilization [59]. (d) Schematics illustrating the cellular interactions involved in the control of bone resorption via the metabolic activity of osteoclasts [3]. Stromal cells (blue) enhance or reduce the activity of osteoclasts (green) through the expression of the ligands RANKL or OPG, respectively. Graphs $(\mathrm{a}-\mathrm{c})$ were adapted and reproduced with permission from Turner [59]. Drawing shown in (d) was adapted and reproduced with permission from Robling et al. [3]

regions within the skeleton by new flawless tissue, thus providing resilience and longevity to the structure.

From an engineering perspective, the process of bone modeling is a remarkable feat. It is analogous to the task of permanently reconstructing and growing the primary structure of a skyscraper using minimal material resources while the building is already loaded with people working in offices at full capacity. How do our bones manage to accomplish such a challenging task? Research on bone modeling suggests that this is achieved by constantly monitoring the local strain within the entire structure and using this information to decide where existing bone should be removed and where new tissue must be deposited. The fact that this occurs through the autonomous action of a large quantity of individual cells without control from a central decision making unit (brain) demonstrates the complexity underlying the adaptive behavior and modeling process of bone.

\subsection{Bone as a mechanostat}

In the $1960 \mathrm{~s}$, bone modeling was proposed by Frost to operate as a closed loop control system 5961 . A wellknown example of such a system is the thermostat used in the kitchen at home to control the temperature of an oven. In this so-called mechanostat model, a negative feedback control system ensures that the level of local mechanical strain in the bone is maintained between two physiologically relevant setpoint values (Figure 8a). The upper setpoint $\left(\varepsilon_{u}\right)$ is defined by the maximum local strain that can be tolerated by bone without undergoing long-term failure, which typically falls within the range $1.5-2.5 \times 10^{-3} 59$. The value defining the lower setpoint $\left(\varepsilon_{l}\right)$ has been suggested to lie between 0.05 and $0.2 \times 10^{-3}$ [59], which is probably a strain level that indicates a sufficient load-bearing contribution of the local tissue to the overall rigidity of the bone. Using this mechanostat model, the adaptive behavior of bone can be described using the language and terms typically employed for dynamic control systems (Figures 8b, c). In a negative feedback closed loop configuration, such a system may comprise a sensor $(\mathrm{S})$ to measure the parameter of interest, a regulator $(\mathrm{R})$ to process the sensing signal, and an effector (E) to keep the parameter of interest within the pre-defined setpoints.

In the case of bone, pre-osteoblastic cells in the marrow and osteocytes embedded in the mineralized tissue have been suggested to act as local mechanical sensors 3 . The role of effectors that change the rigidity of bone by favoring either mineralization or resorption 
processes are predominantly played by osteoblasts and osteoclasts, respectively. During operation of the mechanostat, the local strain $(\varepsilon)$ is the parameter of interest that is constantly monitored by the sensing cells and compared with the lower and upper set point values $\left(\varepsilon_{l}\right.$ and $\left.\varepsilon_{u}\right)$. If the actual strain falls below $\varepsilon_{l}$, the balance between the activity of osteoblasts and osteoclasts is shifted towards local mineral resorption. Instead, if $\varepsilon$ is higher than the upper set point $\left(\varepsilon_{u}\right)$, the balance is changed to promote more bone formation. This negative feedback control mechanism allows the strain to be maintained within a desired interval $\left(\varepsilon_{l} \leq \varepsilon \leq \varepsilon_{u}\right)$ that ensures mechanical stability with minimum weight. Although the mechanostat model does not consider local effects and does not specify the underlying mechanisms that drive the system, it has been successfully used to describe the overall changes in bone rigidity in response to changes in the mechanical loading conditions (Figure 8k). Such a model has also been used to interpret the effect of non-mechanical agents, such as hormones or pharmaceuticals, on bone rigidity and stability. These non-mechanical agents presumably shift the balance between osteoblast and osteoclast activity by changing the set points of the mechanostat [3].

One of the mechanisms that contributes to the observed homeostatic control is the formation of new osteoclasts induced by stromal-derived cells present in the bone marrow (Figure 8d). This process, known as osteoclastogenesis, is regulated by several biomolecules that enable signal processing between cells through specific ligand-receptor interactions. Osteoclastogenesis is presumably favored under low mechanical loading conditions due to the release of RANK ligands (RANKL) by stress-sensitive stromal-derived cells. Such ligands bind to RANK receptors present on the surface of osteoclasts, promoting the metabolic activity of these cells and thus bone resorption. In contrast, higher mechanical stresses cause stromal-derived cells to preferentially secrete OPG, which is another biomolecule that works as a dummy receptor for RANKL. Binding of OPG to RANKL prevents the RANK receptors on the osteoclasts from interacting with the resorptionpromoting ligand. This eventually leads to the programmed death (apoptosis) of osteoclasts and therefore a reduction in bone resorption. Such biochemical signaling process provides a molecular mechanism for the effect of mechanical stress on changes in bone rigidity. In the mechanostat model, the stromal-derived cells play the role of the sensor that monitors the local strain and of the regulator that generates an amplified signal to trigger bone resorption, whereas osteoclasts are the effectors that release chemicals close to the bone surface to dissolve its mineral phase and promote resorption.

\subsection{Osteocyte network and bone remodeling}

While the adaptive behavior and disease conditions of bone can be described in terms of changes in the balance between the metabolic activity of osteoblasts and osteoclasts, osteocytes have been shown to also play a major role in bone dynamics, particularly for homeostasis and the long-term mechanical stability of the tissue. By forming an extensive interconnected network throughout the bone tissue, osteocytes are about 20 times more numerous than osteoblasts and osteoclasts. The openings that host the main body of the osteocytes in the mineralized bone tissue are known as lacunae. Lacunae are connected via numerous small channels called canaliculi, which host long cytoplasmic extensions known as osteocyte processes. This network architecture and the abundance of this cell type is related to the tissue morphogenesis. Osteocytes originate from osteoblasts that are entrapped within the mineralized tissue at the end of the bone formation process. Recent research suggests that the network of osteocytes functions as a sensor that constantly monitors the local strain distribution throughout the tissue. Sensing is hypothesized to be associated with fluid flow in the canaliculi, which might enable mechanotransduction via the build-up of electrical charge or deformation of transmembrane proteins (Figures 9 and 10] 3]. Such a fluid-mediated strain amplification process was put forward to explain the ability of osteocytes to sense strain, after it became clear that the elastic strains developed within the solid phase of bone tissue were too low to induce cellular response.

The sensing capabilities of the osteocyte network is crucial for the remodeling process of bone. Remodeling occurs through the coordinated action of osteoblasts, osteoclasts and other bone cells inside a large agglomerate of cells known as the Basic Multicellular Unit (BMU). Such multicellular units are present on the surface of trabecular bone or embedded within the structure of cortical bone. In cortical bone, the BMU is analogous to a boring machine that excavates bone to remove damaged sites and replace them with new flawless tissue. BMUs are presumably recruited by osteocytes when the local strain level in the tissue exceeds the physiologically relevant set point (Figure 9a). Alternatively, it has also been proposed that the recruitment of BMUs is triggered by cell death caused by micro-cracks that interrupt cell processes of osteocytes. Operating in parallel along the longitudinal axis of the bone, these remarkable cell agglomerates are responsible for the formation of osteons, which are their main structural signature. To move within the tissue and enable bone remodeling, an individual BMU is equipped with osteoclasts at the front and osteoblasts at the rear. This configuration allows osteoclasts to resorb bone in the flawed areas detected by the osteocytes and prompts osteoblasts to lay down new defect-free bone tissue as replacement. All the information required for this orchestrated action to take place is processed and signaled through intricate biochemical pathways, many of which are not yet fully understood (Figure 10. 

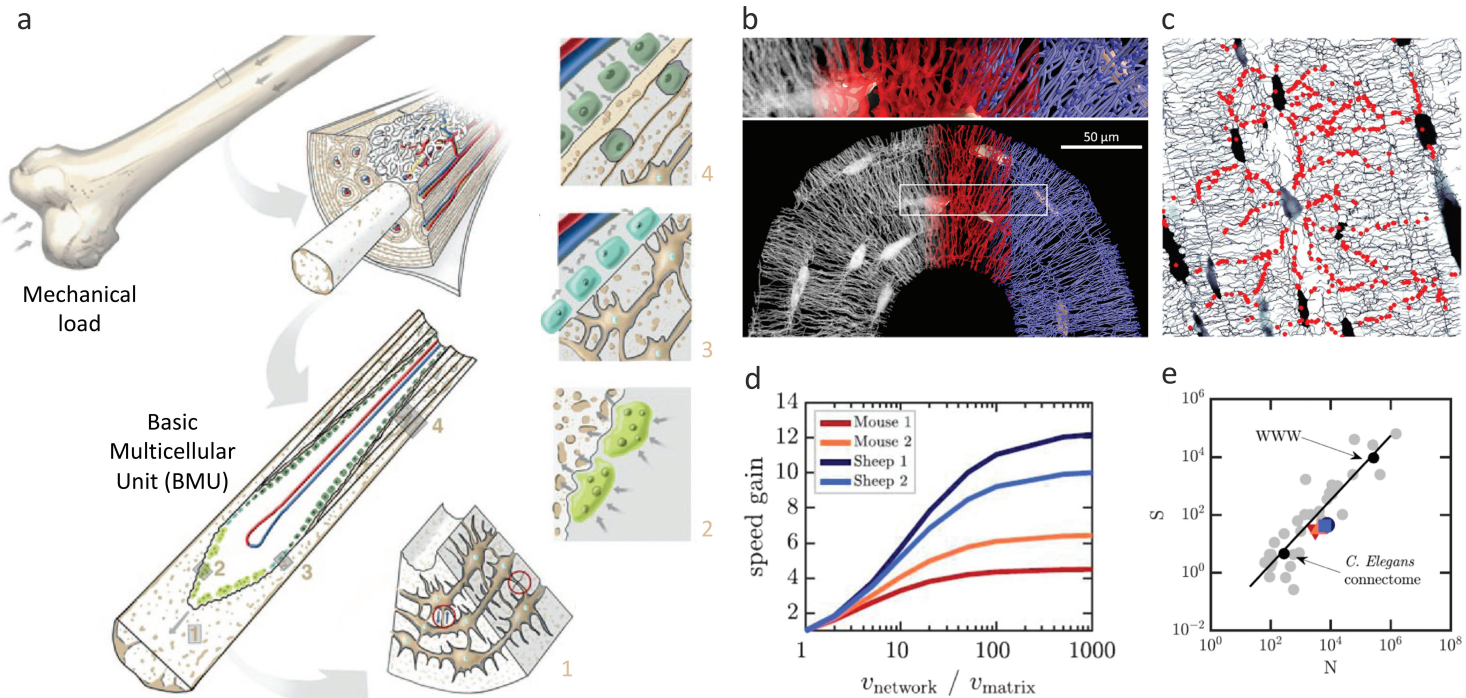

Figure 9: Bone remodeling process. (a) Schematics of the internal structure of the cellular agglomerates, known as Bone Multicellular Units (BMU), responsible for bone tissue remodeling. The network of osteocytes (1), osteoclasts (2), reversal cells (3) and osteoblasts (4) that form the BMU are indicated in the cartoon. Adapted and reproduced with permission from Studart [65]. (b) The osteocyte network shown by a combination of optical microscopy, confocal microscopy and a computer-generated graphical representation. Adapted and reproduced with permission from Weinkamer et al. [63. (c) Selected area of the network depicting (in red) nodes that form "highways" for fast nutrient and signal transmission throughout the tissue. Adapted and reproduced with permission from Weinkamer et al. 63. (d) Expected gain in transmission speed for distinct types of bone depending on the ratio between the transport velocity in the network relative to the bone matrix. Adapted and reproduced with permission from Kollmannsberger et al. [62]. (e) Dependence of the small-worldness index $\mathrm{S}$ on the network size (N) for the osteocyte lacunacanalicular system (colored dots) compared to other real-world networks (black and grey). Adapted and reproduced with permission from Kollmannsberger et al. 62.

The complexity of bone is not limited to the multiple biochemical pathways and associated chemical networks involved in the remodeling process. Remarkably, experimental and modeling studies have shown that the network of interconnected osteocytes also exhibits distinctive features of complex systems [62 64] One of these features involves the notion of a smallworld network. Taking nodes interconnected by links as an abstract model of a network, the small-world characteristic is observed when any two nodes of a large network are separated by only a small number of links [1. This small-world feature has been found in a wide range of self-organized complex systems, including neuron networks in the brain, the world wide web and ecological networks [1. The small-world nature of the osteocyte network is manifested by the presence of a relatively small number of central cells that work as hubs to which many canaliculi are connected (Figure 9p, c).

By enabling the connection of distant nodes via a small number of links, small-world networks facilitate communication while minimizing the energy and costs associated with information processing. In bone, this allows for the supply of nutrients to osteocytes and the transmission of mechano-transduced signals to BMUs with minimum energy input 64. The expected gain in transmission speed through the osteocyte network has been estimated for two types of skeletal tissues: the fibrolamellar bone of sheep and the woven bone of mouse. The more ordered structure of fibrolamellar bone leads to a stronger small-world character, which is expected to accelerate the transportation of nutrients by a factor of 10 compared to the bone matrix without a network. This estimate assumes that diffusion via the canaliculae is 100 times faster than in the bone matrix $\left(v_{\text {network }} / v_{\text {matrix }}=100\right)$. Likewise, the less organized woven architecture leads to a 6 -fold increase in transport speed relative to the matrix (Figure 9d). Compared to other complex networks, the osteocyte network displays a small-world character that lies in between that of the world wide web and the neural network of the model worm C. elegans (Figure 9e). This network connectivity correlates well with the intermediate size of the osteocyte network in comparison to these other examples.

The network of osteocytes is expected to also show another universal feature of small-world networks, namely the trade-off between resilience and vulnerability. On the one hand, the network is resilient against the loss of random nodes with low interconnectivity, which constitute the vast majority in the network. On the other hand, the reliance of the network on a small quantity of essential hubs increases its vulnerability to targeted attacks. The possible effect of this trade-off on the longterm mechanical stability of bone and its behavior under the influence of medication or disease is an open question 


\section{Mechanotransduction and biochemical pathways in bone}

Bone is a complex living material that adapts to environmental conditions, weakening to conserve resources when unused, and strengthening when used often. Cells within bone need to sense and respond to stresses, a task complicated by the inherent stiffness of bone, as forces result in small strains on individual cells. As such, signals generated by the strain require significant amplification. Here, we provide a brief glimpse into some of the molecular events underpinning this adaptive response.

When the bone is compressed by an external force, the fluid in the canalicular network within the mineralized tissue is forced to flow out (Figure 10.1). As the fluid flows past the osteocyte cells, it exerts forces on cell membranes, which induce changes in the protein channels embedded in the membrane. Certain channels begin importing extracellular calcium ions into the osteocytes within 20 seconds of sensing the force. Calcium ions play an important role in many biological processes. In osteocytes, the increase in intracellular calcium leads to the release of adenosine tri-phosphate (ATP) into the extracellular fluid. The ATP binds to receptors on the surface of nearby cells, inducing further changes and the release of other signalling molecules. In this way, the signal generated by the mechanosensory event is propagated and reinforced throughout the tissue. Osteocytes in the network release a range of signalling molecules that eventually reach the osteoblasts, the cell type primarily responsible for the creation of new bone (Figure 10.1b). Furthermore, adjacent cells are connected by gap junctions, allowing signals to pass directly between cells. Osteoblasts are also capable of sensing mechanical forces in similar ways to the osteocytes, and signals from different sources combine and reinforce each other.
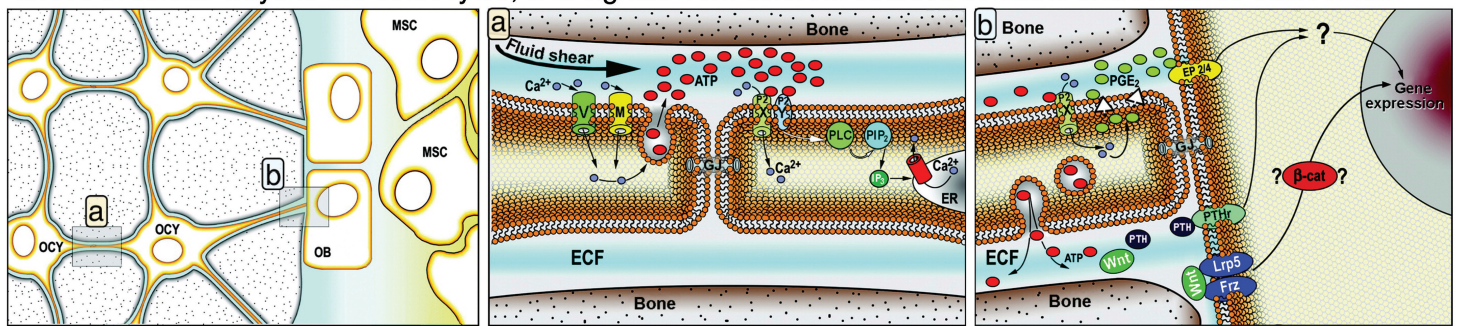

Figure 10.1: Mature bone tissue is composed of a mineralized matrix encasing osteocyte (OCY) cells, interconnected by channels. (a) When the bone is put under stress, the extracellular fluid (ECF) is forced through these channels between the mineralized tissue, leading to shear forces on the osteocyte cell membrane that initiate biochemical signalling processes. (b) Signals released by the osteocytes affect gene regulation within the osteoblasts (OB). The cartoon indicates several of the biomolecules involved in the signalling process. Neighboring cells are also connected by gap junctions (GJ), facilitating direct intercellular communication.

The detection and binding of signaling molecules by receptors on the osteoblast surface initiates an intracellular biochemical cascade involving many actors (Figure 10.2). The Ras, MEKK and MEK proteins are examples of kinase enzymes that take part in this process. Such enzymes chemically modify their target protein by adding a phosphory group to the molecule. This modification, known as phosphorylation, induces a functional change in the target. Each enzyme phosphorylates the next in the series, activating their target and generating a cascade that can amplify the signal at each successive step. The signalling cascades lead to changes in proteins in the osteoblast nucleus, which alter the pattern of gene expression such that collagen production and mineralization-related genes are enhanced. Many of these signal transduction pathways are interconnected through a variety of interactions. The complexity of such signalling networks underlies many favorable emergent properties, such as sensitivity, robustness and noisefiltering. This facilitates reliable signal transduction in a noisy biological environment Many biological networks have complex interconnections, including 'small-world' topologies that exploit the beneficial features of networks.

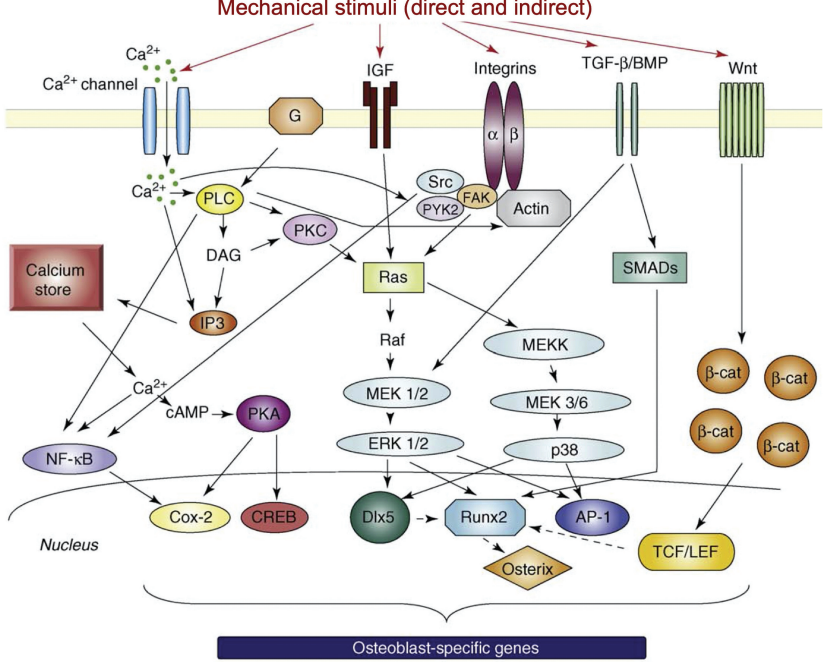

Figure 10.2: The network of mechanosensory signalling pathways inside osteoblast cells. The receptors on the cell membrane at the top of the panel sense either a mechanical force directly, or signalling molecules derived from other mechanosensory events. The receptors then activate downstream signalling molecules, initiating cascades that eventually increase the expression of genes related to bone formation.

This tutorial provides only a glimpse into the complexity underlying the adaptation of bone. There are still many mysteries to this fascinating process that are active topics of research.

Figure 10: Tutorial on bone mechanotransduction [3, 66, 69]. Images are adapted and reproduced with permission from Robling et al. 3] and Papachroni et al. 67. 
of direct relevance for the understanding of this complex material and for the possible identification of better protocols for the medical diagnosis and treatment of injured bone.

\section{Conclusions}

Bone is a fascinating material that combines the living nature of a biological tissue with mechanical properties that are quite unusual in view of its weak and soft chemical constituents. Based on the way information is processed during growth and maintenance of the tissue, bone can be understood as a complex system in which properties emerge from the interactions of individual cells rather than the top-down control by a central processing unit. This leads to universal features that are also found in other complex systems, such as self-organization, hierarchy and adaptive behavior. Selforganization is driven by the activities of living cells, which explore supramolecular, covalent and ionic bonds to assemble collagen molecules and hydroxyapatite platelets in remarkably ordered mineralized fibrils. The arrangement of such fibrils at multiple length scales results in hierarchical structures that are simultaneously stiff and fracture resistant, two antagonistic properties that are seldom found in engineering materials. Through the concerted action of living cells, the stiff and tough structure of bone is able to grow and adapt itself to mechanical stimuli from the environment. From an engineering perspective, this set of properties makes bone quite a remarkable material that serve as an inspiration for the development of future lightweight, sustainable and durable load-bearing structures. The recent finding that osteocytes interconnected throughout bone display network properties analogous to those found in neural networks shows how much there is still to be explored in this fascinating material. Further understanding of bone as a complex system will require expertise from multiple disciplines, which makes this material also an exciting platform for collaborative teaching and research.

\section{Author Contributions}

T.M and R.L have equally contributed to this manuscript and are therefore jointly first authors. All authors contributed to the conceptualization, drafting, discussion and revision of the manuscript. R.L. compiled information about collagen synthesis and bone mineralization models, discussed the underlying self-organization process and drafted the tutorial panel describing the supramolecular chemistry of collagen. T.M. reviewed the hierarchical structure of bone and the spectroscopic characterization of osteons, revisited the mechanical properties of bone and drafted the tutorial on fracture mechanics of materials. A.R.S. worked on the overall structure of the manuscript, reviewed the adaptive behavior and the remodeling processes of bone, and discussed the properties of the osteocyte network. A.K. wrote the tutorial panel about mechanotransduction and biochemical pathways in bone, and critically assessed the cellular mechanisms underlying bone remodeling.

\section{Acknowledgement}

We thank Dr. Florian Bouville (Imperial College, UK) for the kind permission to reproduce his artwork shown in Figure 1 $(\mathrm{a}, \mathrm{b})$.

\section{References}

[1] M. Mitchell, Complexity: A Guided Tour (Oxford University Press, Inc., 2009).

[2] H. Haapasalo, S. Kontulainen, H. Sievänen, P. Kannus, M. Järvinen and I. Vuori, Bone 27, 351 (2000).

[3] A.G. Robling, A.B. Castillo and C.H. Turner, Annual Review of Biomedical Engineering 8, 455 (2006).

[4] J. Wolff, The Law of Bone Remodelling (Translated by P. Maquet, and R. Furlong). (Springer Berlin Heidelberg, 1986).

[5] H.D. Espinosa, J.E. Rim, F. Barthelat and M.J. Buehler, Prog. Mater. Sci. 54, 1059 (2009).

[6] P. Garnero, Calcified Tissue International 97, 229 (2015).

[7] O.G. Andriotis, S.W. Chang, M. Vanleene, P.H. Howarth, D.E. Davies, S.J. Shefelbine, M.J. Buehler and P.J. Thurner, Journal of The Royal Society Interface 12, 20150701 (2015)

[8] G. Plopper, Principles of Cell Biology (Jones \& Bartlett Learning, Burlington, MA, 2012).

[9] J.U. Adams, Essentials of Cell Biology (NPG Education, Cambridge, MA, 2010).

[10] D. Parisuthiman, Y. Mochida, W.R. Duarte and M. Yamauchi, Journal of Bone and Mineral Research 20, 1878 (2005).

[11] M. Terajima, I. Perdivara, M. Sricholpech, Y. Deguchi, N. Pleshko, K.B. Tomer and M. Yamauchi, J. Biol. Chem. 289, 22636 (2014).

[12] H.J. Jürgensen, D.H. Madsen, S. Ingvarsen, M.C. Melander, H. Gårdsvoll, L. Patthy, L.H. Engelholm and N. Behrendt, J. Biol. Chem. 286, 32736 (2011).

[13] J.F. Lees and N.J. Bulleid, J. Biol. Chem. 269, 24354 (1994).

[14] P.L. Connerly, Nature Education 3, 60 (2010).

[15] A.L. Boyle and D.N. Woolfson, in Supramolecular Chemistry: From Molecules to Nanomaterials, edited by P. A. Gale (John Wiley \& Sons, Ltd., Hoboken, NJ, 2012).

[16] D.B. Varshey, J.R.G. Sander, T. Frišcic and L.R. MacGillivray, in Supramolecular Chemistry: from Molecules to Nanomaterials, edited by J. W. Steed, P.A. Gale. (John Wiley \& Sons, Ltd, Hoboken, NJ, 2012).

[17] J. Bella, Biochem. J 473, 1001 (2016).

[18] J.A. Fallas, J. Dong, Y.J. Tao and J.D. Hartgerink, J. Biol. Chem. 287, 8039 (2012).

[19] S. Zhu, Q. Yuan, T. Yin, J. You, Z. Gu, S. Xiong and Y. $\mathrm{Hu}$, Journal of Materials Chemistry B 6, 2650 (2018). 
[20] https://commons.wikimedia.org/wiki/File:Collagen_(tri ple_helix_protein_with_schematic_ribbons).jpg, accessed in Dec. 2020.

[21] F. Melsen and L. Mosekilde, Acta Pathologica Microbiologica Scandinavica Section A Pathology 88A, 83 (1980).

[22] M.J. Glimcher, H. Muir, A. Miller, D.C. Phillips and R.J.P. Williams, Philosophical Transactions of the Royal Society of London. B, Biological Sciences 304, 479 (1984).

[23] H.C. Anderson, Clin Orthop Relat Res 314, 266 (1995).

[24] J. Mahamid, A. Sharir, L. Addadi and S. Weiner, Proceedings of the National Academy of Sciences 105, 12748 (2008).

[25] L. Addadi and S. Weiner, Phys. Scr. 89, 098003 (2014).

[26] F. Nudelman, A.J. Lausch, N. Sommerdijk and E.D. Sone, Journal of Structural Biology 183, 258 (2013).

[27] J.J. Jasielec, R. Filipek, K. Dołowy and A. Lewenstam, Membranes 10, 81 (2020).

[28] J.H. Martin and J.L. Matthews, Clin Orthop Relat Res 68, 273 (1970).

[29] L.V. Sutfin, M.E. Holtrop and R.E. Ogilvie, Science 174, 947 (1971).

[30] S. Boonrungsiman, E. Gentleman, R. Carzaniga, N.D. Evans, D.W. McComb, A.E. Porter and M.M. Stevens, Proceedings of the National Academy of Sciences 109, 14170 (2012).

[31] J. Mahamid, A. Sharir, D. Gur, E. Zelzer, L. Addadi and S. Weiner, Journal of Structural Biology 174, 527 (2011).

[32] T. Hasegawa, Histochemistry and Cell Biology 149, 289 (2018).

[33] H. Colfen, Nat. Mater. 9, 960 (2010).

[34] A. Vei and J.R. Dorvee, Calcified Tissue International 93, 307 (2013).

[35] S. Omelon, J. Georgiou, Z.J. Henneman, L.M. Wise, B. Sukhu, T. Hunt, C. Wynnyckyj, D. Holmyard, R. Bielecki and M.D. Grynpas, PLOS ONE 4, e5634 (2009).

[36] F.S. Sayegh, G.C. Solomon and R.W. Davis, Clinical Orthopaedics and Related Research $\AA$ 99, 267 (1974).

[37] S. Weiner and L. Addadi, Annual Review of Materials Research 41, 21 (2011).

[38] W.J. Jin, S. Q. Jiang, H.H. Pan and R.K. Tang, Crystals 8, (2018).

[39] Z.J. Xu, Y. Yang, W.L. Zhao, Z.Q. Wang, W.J. Landis, Q. Cui and N. Sahai, Biomaterials 39, 59 (2015).

[40] F. Nudelman, K. Pieterse, A. George, P.H.H. Bomans, H. Friedrich, L.J. Brylka, P.A.J. Hilbers, G. de With and N. Sommerdijk, Nat. Mater. 9, 1004 (2010).

[41] U.G.K. Wegst, H. Bai, E. Saiz, A.P. Tomsia and R.O. Ritchie, Nat. Mater. 14, 23 (2015).

[42] S.M. Ott, American Journal of Nephrology 47, 373 (2018).

[43] S. Schrof, P. Varga, L. Galvis, K. Raum and A. Masic, Journal of Structural Biology 187, 266 (2014).

[44] M.B. Schaffler, D.B. Burr and R.G. Frederickson, The Anatomical Record 217, 223 (1987).

[45] X.N. Dong, X. Zhang and X.E. Guo, Mech Chem Biosyst 2, 63 (2005).
[46] D.B. Burr, M.B. Schaffler and R.G. Frederickson, Journal of Biomechanics 21, 939 (1988).

[47] G. Marotti, M. Ferretti and C. Palumbo, Journal of Morphology 274, 543 (2013).

[48] M.M. Giraud-Guille, Calcified Tissue International 42, 167 (1988).

[49] S. Weiner, W. Traub and H.D. Wagner, Journal of Structural Biology 126, 241 (1999).

[50] W. Wagermaier, H.S. Gupta, A. Gourrier, M. Burghammer, P. Roschger P. Fratzl, Biointerphases 1, 1 (2006).

[51] A. Ascenzi, E. Bonucci, P. Generali, A. Ripamonti and N. Roveri, Calcified Tissue International 29, 101 (1979).

[52] M.E. Launey, M.J. Buehler and R.O. Ritchie, Annual Review of Materials Research 40, 25 (2010).

[53] S. Pabisch, W. Wagermaier, T. Zander, C. Li and P. Fratzl, Methods Enzymol 532, 391 (2013).

[54] A. Masic, L. Bertinetti, R. Schuetz, L. Galvis, N. Timofeeva, J. W. Dunlop, J. Seto, M. A. Hartmann and P. Fratzl, Biomacromolecules 12, 3989 (2011).

[55] H.S. Gupta, J. Seto, W. Wagermaier, P. Zaslansky, P. Boesecke and P. Fratzl, Proceedings of the National Academy of Sciences of the United States of America 103, 17741 (2006)

[56] R.O. Ritchie, Nat. Mater. 10, 817 (2011).

[57] K.J. Koester, J.W. Ager and R.O. Ritchie, Nat. Mater. 7, 672 (2008).

[58] A. Bhaduri, Fracture (Springer, Singapore, 2018), vol. 264, p. 758.

[59] C.H. Turner, Bone 12, 203 (1991).

[60] H.M. Frost, Anatomical Record 219, 1 (1987).

[61] H.M. Frost, Bone Remodelling Dynamics (Charles C Thomas, 1963).

[62] P. Kollmannsberger, M. Kerschnitzki, F. Repp, W. Wagermaier, R. Weinkamer and P. Fratzl, New Journal of Physics 19, 073019 (2017).

[63] R. Weinkamer, P. Kollmannsberger and P. Fratzl, Current Osteoporosis Reports 17, 186 (2019).

[64] M. Kerschnitzki, P. Kollmannsberger, M. Burghammer, G.N. Duda, R. Weinkamer, W. Wagermaier and P. Fratzl, Journal of Bone and Mineral Research 28, 1837 (2013).

[65] A.R. Studart, Angewandte Chemie-International Edition 54, 3400 (2015).

[66] C.T. Hung, F.D. Allen, S.R. Pollack and C.T. Brighton, Journal of Biomechanics 29, 1403 (1996).

[67] K.K. Papachroni, D.N. Karatzas, K.A. Papavassiliou, E.K. Basdra and A.G. Papavassiliou, Trends in Molecular Medicine 15, 208 (2009).

[68] E.U. Azeloglu and R. Iyengar, Cold Spring Harb Perspect Biol 7, a005934 (2015).

[69] A. Wagner and D.A. Fell, Proceedings. Biological sciences 268, 1803 (2001). 